\title{
Assay for UDPglucose 6-dehydrogenase in phosphate-starved cells: gene tuaD of Bacillus subtilis 168 encodes the UDPglucose 6-dehydrogenase involved in teichuronic acid synthesis
}

\author{
Marco Pagni, Vladimir Lazarevic, Blazenka Soldo and Dimitri Karamata
}

Institut de génétique et de biologie microbiennes, Rue César-Roux 19, $\mathrm{CH}-1005$ Lausanne, Switzerland
Author for correspondence: Dimitri Karamata. Tel: +4121 3206075. Fax: +41213206078. e-mail : dimitri.karamata@igbm.unil.ch
Keywords: UDPglucose 6-dehydrogenase, enzyme assay, Bacillus subtilis, teichuronic acid

\section{INTRODUCTION}

UDPglucose 6-dehydrogenase (UDPG-DH, EC 1.1.1.22) converts UDPglucose (UDPG) into UDPglucuronic acid (UDPGUA). The latter, as well as UDP- $N$-acetylgalactosamine, are precursors of teichuronic acid of Bacillus licheniformis 94 (Hughes, 1970; Janczura et al., 1961) and Bacillus subtilis W23 (Wright \& Heckel, 1975). Ellwood \& Tempest (1969) and Kruyssen et al. (1980) have incorporated UDPG-DH into the hypothetical metabolic pathway leading to teichuronic acid. Although UDPG-DH activity was assayed in cell-free extracts from many organisms, its activity was never conclusively established in any

Abbreviations: UDPG, uridine $5^{\prime}$-diphosphoglucose (UDPglucose); UDPGUA, uridine 5'-diphosphoglucuronic acid (UDPglucuronic acid); UDPG-DH, UDPglucose 6-dehydrogenase.
Bacillus species. Indeed, the only evidence in favour of UDPG-DH activity in a cell-free extract of B. subtilis 168 trp was obtained by an indirect (Rosenberger, 1976) and possibly inappropriate method (see below).

Analysis of recently obtained nucleotide sequences of $B$. subtilis 168 allowed the identification of an operon consisting of eight genes, tuaA-H, involved in the synthesis of teichuronic acid (Soldo et al., 1999). The product of tuaD exhibits a high similarity with proteins belonging to the UDPglucose/GDPmannose dehydrogenase family, as well as with the products of $y w q F$ and $y t c A$, two B. subtilis 168 genes, located in the same chromosomal region as the tua operon.

In this paper, we report (i) a reliable method for preparing cell-free extracts allowing a spectrophotometric assay of UDPG-DH activity, (ii) a demonstration that tuaD of $B$. subtilis 168 is the structural gene of UDPG-DH, a conclusion that could not have been 
reached from sequence comparison, and (iii) that TuaD is responsible for the synthesis of the entire UDPGUA pool in phosphate-starved $B$. subtilis.

\section{METHODS}

Bacterial strains. Strain 168 , the $B$. subtilis reference strain, whose genome nucleotide sequence was reported by Kunst et al. (1997), was used as the parent strain for the construction of mutants deficient in teichuronic acid synthesis. Strains L16050 tuaC, L16051 tuaD-H, L16054 tuaD and L16071 tuaG were obtained by Campbell insertion of plasmids constructed with pMTL20EC (Chambers et al., 1988), a vector conferring chloramphenicol resistance and inserts corresponding to SubtiList (Moszer et al., 1995) base numbers 36556643654953 , 3654128-3653562, 3654439-3654016 and 36508603650480, respectively. Strains L16050 tuaC, L16054 tuaD and L16071 tuaG are able to express tua genes located downstream of the inactivated ones, owing to the presence of a plasmidborne promoter. Details on the molecular sequence of the tua operon and on the inability of the mutants to incorporate teichuronic acids in their cell wall can be found elsewhere (Soldo et al., 1999).

Growth conditions. Wild-type and mutants were grown in low-phosphate medium (Grant, 1979) supplemented with $300 \mu \mathrm{M}$ inorganic phosphate. For tua-deficient mutants, $3 \mu \mathrm{g}$ chloramphenicol $\mathrm{ml}^{-1}$ was added to prevent reversion to $t u a^{+}$, due to plasmid excision. Exponentially growing cells at a low density were used to inoculate $1 \mathrm{l}$ cultures in 51 flasks which were further incubated, by shaking at $37^{\circ} \mathrm{C}$, and harvested about $12 \mathrm{~h}$ after the onset of phosphate limitation, which was assessed as described by Grant (1979). Cells at a concentration of $0.4 \mathrm{~g}$ dry weight $\mathrm{l}^{-1}$ were pelleted, washed once with icecold bidistilled water and stored frozen at $-20^{\circ} \mathrm{C}$.

Preparation and chromatography of nucleotide precursors. A frozen cell pellet corresponding to $330 \mathrm{ml}$ culture was thawed in $3 \mathrm{ml}$ boiling water, incubated for $15 \mathrm{~min}$ at $100^{\circ} \mathrm{C}$ and centrifuged. The supernatant was mixed with $1 \mathrm{ml}$ activated charcoal GR (Merck) that had been washed with acetic acid and ethanolic ammonia, dried and resuspended in water at $50 \mathrm{mg} \mathrm{ml}^{-1}$. The mixture was incubated for $1 \mathrm{~h}$ at room temperature with gentle shaking. Charcoal was collected by centrifugation, washed three times with $5 \mathrm{ml} 100 \mathrm{mM}$ acetic acid and extracted twice for $60 \mathrm{~min}$ at $70^{\circ} \mathrm{C}$ with $2 \mathrm{ml} 25 \mathrm{mM}$ $\mathrm{NH}_{3}$ in $50 \%$ ethanol. The two extracts were pooled, frozen, lyophilized and resuspended in $1 \mathrm{ml}$ water to yield a crude preparation of nucleotide precursors.

A $500 \mu$ portion of the nucleotide precursors preparation was diluted to $2 \mathrm{ml}$ with $10 \mathrm{mM}$ ammonium acetate, filtered through the $0.22 \mu \mathrm{m}$ membrane filter to remove charcoal particles and loaded onto a strong anion-exchanger FPLC column (MonoQ HR 5/5, Pharmacia) preconditioned in $10 \mathrm{mM}$ ammonium acetate. Elution at $0.5 \mathrm{ml} \mathrm{min}^{-1}$ with a $50 \mathrm{~min}$ linear concentration gradient was performed with $1 \mathrm{M}$ ammonium acetate as the second buffer and monitored by absorbance at $275 \mathrm{~nm}$. The peak corresponding to UDPGUA was identified by (i) its retention time, (ii) its UV absorption spectrum and (iii) the Blumenkrantz \& Asboe-Hansen (1973) assay for uronic acid, for which the peak material reacted positively, providing an amount of UDPGUA within $10 \%$ of that deduced from the absorbance spectrum. The UDPGUA peak was integrated and quantified by comparison to a reference curve obtained by chromatography of known amounts of commercial UDPGUA. To determine the yield of the UDPGUA preparation, known amounts of UDPGUA were added to samples of the crude extract of nucleotide precursors before charcoal adsorption. Purification and chromatography resulted in a $25-45 \%$ loss of the internal standard. UDPGUA amounts in unspiked samples were corrected accordingly. The method was capable of detecting $5 \mathrm{nmol}$ UDPGUA.

Preparation of the cell-free extract. A thawed pellet corresponding to a $330 \mathrm{ml}$ sample of phosphate-limited culture was resuspended in $8 \mathrm{ml}$ protoplasting buffer $(50 \mathrm{mM}$ Tris/ $\mathrm{HCl} \mathrm{pH} \mathrm{8.6;} 100 \mathrm{mM} \mathrm{KCl} ; 25 \%$, w/v, sucrose; 1 g lysozyme $\mathrm{I}^{-1}$ ) and incubated for $20 \mathrm{~min}$ at $37^{\circ} \mathrm{C}$. The sample, cooled on ice, was subsequently processed at $4{ }^{\circ} \mathrm{C}$. Protoplasts were harvested by centrifugation, washed once with $8 \mathrm{ml}$ lysozymefree protoplasting buffer and lysed by resuspension in $2 \mathrm{ml}$ lysis buffer ( $50 \mathrm{mM}$ potassium phosphate pH 6.8, $100 \mathrm{mM}$ $\mathrm{KCl}, 1 \mathrm{mM}$ EDTA, $0 \cdot 1 \mathrm{mM}$ PMSF). The lysate was homogenized by a brief ultrasonication and centrifuged for $30 \mathrm{~min}$ at $100000 \mathrm{~g}$. Finally, the supernatant was dialysed overnight against $50 \mathrm{mM}$ potassium phosphate $\mathrm{pH} 6.8,100 \mathrm{mM} \mathrm{KCl}$, $1 \mathrm{mM}$ EDTA, $1 \mathrm{mM}$ DTT, $0.1 \%$ Triton X-100, in a Slide-ALyzer cassette (molecular mass cut-off $10000 \mathrm{Da}$; Pierce). The presence of Triton X-100 and DTT, associated with slightly higher activity recovery, was not essential.

UDPG-DH assay. The dialysed cell-free extract $(25 \mu \mathrm{l})$ was diluted with $175 \mu \mathrm{l} 500 \mathrm{mM}$ glycylglycine/ $\mathrm{NaOH} \mathrm{pH} 9 \cdot 2$, to which $200 \mu \mathrm{l} 10 \mathrm{mM} \mathrm{NAD}{ }^{+}$and $10 \mathrm{mM}$ UDPG, dissolved in the same buffer, were added. The sample was placed in a thermostated 8-cell changer of a Perkin-Elmer spectrophotometer at $37{ }^{\circ} \mathrm{C}$ and absorbance at $340 \mathrm{~nm}$ was recorded at $1 \mathrm{~min}$ intervals for $30 \mathrm{~min}$. A control sample without UDPG was run in parallel.

The stoichiometry of NADH and UDPGUA production was determined in a $2.5 \mathrm{ml}$ reaction mixture from strain $\mathrm{L} 16071$ tuaG characterized by a high level of UDPG-DH. The absorbance spectrum of the assay mixture was periodically recorded to quantify $\mathrm{NADH}$. At regular intervals of time, $400 \mu \mathrm{l}$ aliquots were removed, mixed with $1.6 \mathrm{ml}$ ethanol, kept on ice for $1 \mathrm{~h}$ and centrifuged. The supernatant was collected, lyophilized, resuspended in $10 \mathrm{mM}$ ammonium acetate and chromatographed to quantify UDPGUA as described above.

Other methods. Protein content was assayed with the BCA reagent (Pierce) using bovine serum albumin as standard. UDPG was assayed enzymically with beef liver UDPG-DH (Boehringer Mannheim) according to Strominger et al. (1957). Phosphoglucomutase was assayed according to Forsberg $e t$ al. (1973). Glucose-6-phosphate dehydrogenase was assayed according to Ujita \& Kimura (1982) using $\mathrm{NAD}^{+}$as electron acceptor. Nucleoside diphosphate sugar hydrolase (Mauck \& Glaser, 1970) was detected on isoelectric focusing gels by the direct phosphodiesterase staining method of Némoz et al. (1983) using UDPG as substrate.

\section{RESULTS AND DISCUSSION}

UDPG-DH is required for the synthesis of UDPGUA, one of the precursors of teichuronic acid. In B. subtilis, the synthesis of the latter polymer (Soldo et al., 1999) is induced under conditions of phosphate limitation. However, it is well known that phosphate starvation leads to induction of the synthesis of several phosphatases, phosphodiesterases and nucleases. The parallel appearance of these hydrolytic enzymes may significantly complicate assays for enzymes involved in 


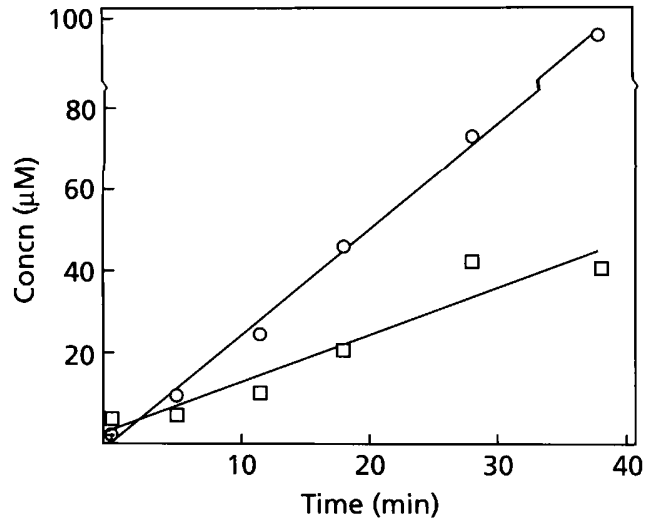

Fig. 1. Assay of UDPG-DH activity in the cell-free extract of strain L16071 tuaG. Kinetics of appearance of NADH $(O)$ and UDPGUA ( $\square$ ) in the reaction mixture.

nucleotide-diphosphate-sugar metabolism through rapid nucleotide degradation. The report of Merchante et al. (1995), strongly suggesting the existence of a $B$. subtilis periplasmic fraction containing these hydrolytic enzymes, offers a possibility to devise reliable assays by avoiding the mixing of the periplasmic fraction with the relevant cytoplasmic fraction. The advantages of the periplasm/cytoplasm separation are illustrated by the straightforward spectrometric assay for UDPG-DH described below.

\section{UDPG-DH assay in B. subtilis}

Cell-free extracts were obtained from cells grown as a batch culture in a low-phosphate medium. Twelve hours after the onset of phosphate limitation, cells were harvested, washed and protoplasted. Protoplasts were washed and disrupted. Membrane debris was removed by ultracentrifugation and the enzyme-containing supernatant was dialysed. UDPG-DH activity, monitored by $\mathrm{NAD}^{+}$reduction, was detected only when UDPG was added to the assay mixture. $\mathrm{NAD}^{+}$reduction in the presence of UDPG increased linearly until the $A_{340}$ reached a value of 1 . The rate of NADH formation decreased subsequently, possibly due to an inhibitory effect of the produced NADH (Ordman \& Kirkwood, 1977). Indeed, addition of $40 \mu \mathrm{M} \mathrm{NADH}$ to the reaction mixture led to a loss of over $90 \%$ of the UDPG-DH activity. Quantification of NADH and UDPGUA, simultaneously accumulating in the reaction mixture, provided a ratio of $2 \cdot 25 \mathrm{NAD}^{+}$reduced per UDPG oxidized, as calculated from the slopes of regression lines (Fig. 1), in good agreement with the expected stoichiometrical coefficient of 2. These observations established that $\mathrm{NAD}^{+}$reduction in the assay is essentially due to the activity of UDPG-DH, which was stable for at least $24 \mathrm{~h}$ in extracts stored on ice.

Protoplasting and removal of the periplasmic fraction was essential for the detection of UDPG-DH activity. Cell-free extracts obtained from sonicated or French- press-broken cells exhibited no UDPG-DH activity in the spectrophotometric assay. It should, however, be pointed out that complete protoplasting was unnecessary. Indeed, even a very limited lysozyme treatment - up to 5 min incubation in osmotically stabilized medium was sufficient for the release of the periplasmic fraction (H. M. Pooley, unpublished). Observations of Merchante et al. (1995), Mauck \& Glaser (1970) and M. Pagni (unpublished) revealed that, compared with the cytoplasmic fraction, phosphodiesterases of the periplasmic fraction are more diverse and their specific activity is at least 10 times higher. The final purification step, i.e. the dialysis of the ultracentrifugation supernatant, was also crucial for the recovery of UDPG-DH activity, which is hardly detectable in undialysed extract. The effect of dialysis might be accounted for by the removal of low-molecular-mass inhibitory compounds. For example, NADH and UDPxylose have been reported to inhibit the beef UDPG-DH (Zalitis \& Feingold, 1969) and, as shown above, NADH was inhibitory to the $B$. subtilis enzyme.

The only previously reported UDPG-DH assay in $B$. subtilis (Rosenberger, 1976) employed cell-free extracts obtained by breaking cells with a French press. Without dialysis, these extracts were assayed for UDPG-DH activity with the radioassay of Davies \& Dickinson (1972). The specificity of this radioassay, established on germinating lily pollen, was never confirmed for Bacillus extracts. The results obtained by Rosenberger (1976) with $B$. subtilis extracts are incompatible with our failure to detect UDPG-DH activity in undialysed extracts obtained with a French press. However, we believe that the activity obtained with this radioassay was an artefact most likely due to contamination of the cell-free extract by the nucleoside-diphosphate-sugar hydrolase activity originating from the periplasmic fraction. Indeed, the latter enzyme was shown to liberate glucose 1-phosphate from UDPG (Mauck \& Glaser, 1970; see Methods). The highly active cytoplasmic phosphoglucomutase and the $\left(\mathrm{NAD}^{+}\right.$-reducing $)$glucose6-phosphate 1-dehydrogenase (Ujita \& Kimura, 1982), whose presence in the extracts was confirmed (M. Pagni, unpublished), would, together with enzymes inherent to the Davies \& Dickinson assay, eventually convert $\left[{ }^{14} \mathrm{C}\right]$ glucose 1-phosphate into $\left[{ }^{14} \mathrm{C}\right]$ gluconate, an acidic sugar, most probably indistinguishable from glucuronate by the paper chromatographic method of Davies \& Dickinson (1972).

\section{Sequence comparison and analysis of tua-deficient strains}

Inspection of the B. subtilis 168 genome sequence (Kunst et al., 1997) yields three genes - tuaD, $y t c A$ and $y w q F-$ which encode enzymes belonging to the UDPglucose/ GDPmannose dehydrogenase family. At the protein level, TuaD exhibits $45 \%$ and $51 \%$ identities with YtcA and YwqF, respectively. A search for similar proteins in the release 35 of the SWISS-PROT database yielded nine proteins with BLAST scores to either of TuaD, YtcA or 
Table 1. UDPG-DH activity and UDPGUA precursor pools in tua-deficient strains, and in B. subtilis 168

Samples from batch cultures were collected $12 \mathrm{~h}$ after the onset of phosphate limitation.

\begin{tabular}{|llcc|}
\hline Strain & Genotype* $^{*}$ & UDPG-DH $\dagger$ & UDPGUA $(\mu \mathrm{M}) \neq$ \\
\hline 168 & Tua $^{+}$ & $2 \cdot 8$ & 203 \\
L16050 & tuaCS $^{\prime}$ & $1 \cdot 0$ & 60 \\
L16051 & tuaD-H & $<0 \cdot 1 \|$ & $<3 \|$ \\
L16054 & tuaD $\mathbb{}$ & $<0 \cdot 1 \|$ & $<3 \|$ \\
L16071 & tuaG $\mathbb{7}$ & $7 \cdot 3$ & 325 \\
\hline
\end{tabular}

* Only markers relevant to teichuronic acid synthesis are indicated.

$\dagger \mu$ mol UDPG oxidized $\min ^{-1}$ (g protein $)^{-1}$.

‡Concentration in a $1 \mathrm{ml}$ solution containing nucleotide precursors extracted from a $330 \mathrm{ml}$ culture.

$\$$ Genes distal to the inactivated one are expressed from the constitutive plasmid-borne promoter.

$\|$ Below the detection threshold.

YwqF of above 400, an arbitrarily chosen figure. Although all were members of the UDPglucose/ GDPmannose dehydrogenase family, unambiguous biochemical identification of their substrates has been achieved for only two of them, i.e. KfDD, a UDPG-DH of Escherichia coli (Petit et al., 1995) and AlgD, a GDPmannose dehydrogenase, of Azotobacter vinelandii (Campos et al., 1996). Further sequence analyses multiple alignments of sequences and computation of their pairwise distances - revealed that the distance between $\mathrm{KfiD}$ and $\mathrm{AlgD}$ is roughly comparable to that separating the three $B$. subtilis dehydrogenases, precluding the assignment of a specific substrate to any of the putative dehydrogenases TuaD, YtcA or YwqF. However, since tuaD belongs to an operon shown to be involved in teichuronic acid synthesis (Soldo et al., 1999), it is likely that it encodes the UDPG-DH required for teichuronic acid synthesis. Therefore, several tuaDdeficient mutants were examined for UDPG-DH activity as well as for the accumulation of UDPG and UDPGUA.

Data presented in Table 1 show that specific inactivation of tuaD, achieved by insertional mutagenesis, is accompanied by the complete loss of UDPG-DH activity. Under the growth conditions used, this unambiguously identifies the product of gene tuaD as UDPG-DH and indicates that the products of neither of the other genes with sequence homology are involved in the oxidation of UDPG under phosphate-limiting conditions. To ascertain that UDPG is the in vivo substrate of UDPGDH in B. subtilis 168 and to examine the effect of UDPGDH deficiency on the cytoplasmic pools of UDPG and UDPGUA, we have measured the latter compounds in all tuaD-deficient strains, as well as in the parent strain. UDPGUA was present in $t u a D^{+}$strains and absent in tuaD strains L16051 tuaD-H and L16054 tuaD (Fig. 2,

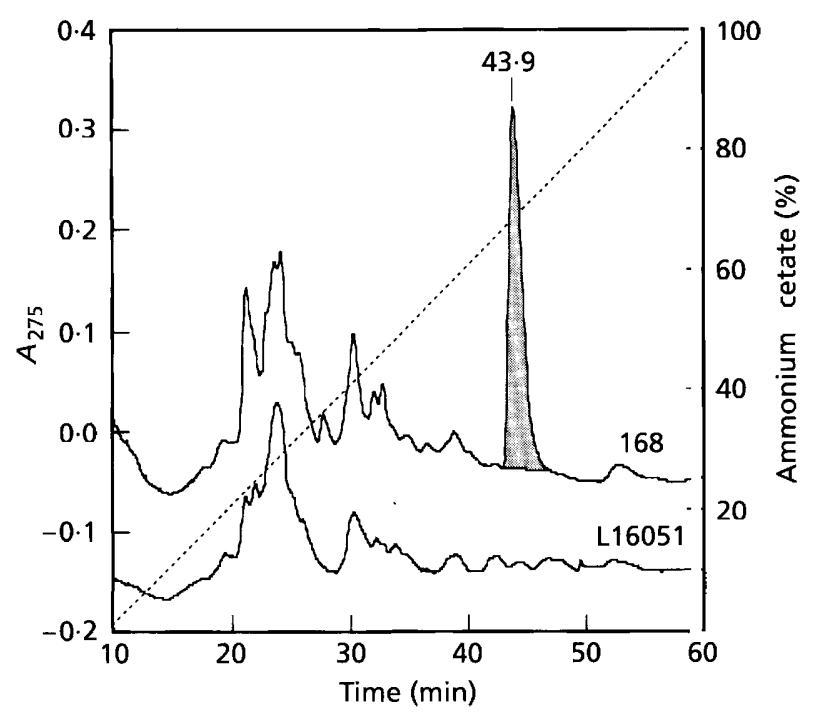

Fig. 2. FPLC of nucleotide precursors extracted from $B$. subtilis 168 and mutant L16051 tuaD-H on a MonoQ column. Elution profiles as detected by absorbance at $275 \mathrm{~nm}(-)$ using a linear concentration gradient of $1 \mathrm{M}$ ammonium acetate (----). The shaded peak corresponds to UDPGUA.

Table 1), confirming that $\mathrm{TuaD}$ is responsible for UDPGUA production during phosphate-limited growth. Enzymic quantification of UDPG in crude nucleotideprecursor preparations of the five examined strains did not reveal a significant accumulation of UDPG (not presented). The measured amounts were low, an order of magnitude lower than those of UDPGUA in strain 168. The apparent absence of UDPG accumulation in tuaD mutants may be accounted for by its channelling into other metabolic pathways, or by feedback inhibition of its synthesis.

\section{ACKNOWLEDGEMENTS}

We would like to thank Patricia Scuderi for skilful technical assistance. The financial support of the Fonds National Suisse de la Recherche Scientifique (grant no. 31-43632.95) is gratefully acknowledged.

\section{REFERENCES}

Blumenkrantz, N. \& Asboe-Hansen, G. (1973). New method for quantitative determination of uronic acids. Anal Biochem 54, 484-489.

Campos, M.-E., Martìnez-Salazar, J. M., Lloret, L., Moreno, S., Núñez, C., Espín, G. \& Soberón-Chávez, G. (1996). Characterization of the gene coding for GDP-mannose dehydrogenase (algD) from Azotobacter vinelandii. J Bacteriol 178, 1793-1799.

Chambers, S. P., Prior, S. E., Barstow, D. A. \& Minton, N. P. (1988). The PMTL nic cloning vectors. I. Improved pUC polylinker region to facilitate the use of sonicated DNA for nucleotide sequencing. Gene 68, 139-149.

Davies, M. D. \& Dickinson, D. B. (1972). Radiochemical assay for UDP-glucose dehydrogenase. Anal Biochem 47, 209-217. 
Ellwood, D. C. \& Tempest, D. W. (1969). Control of teichoic acid and teichuronic acid biosyntheses in chemostat cultures of Bacillus subtilis var. niger. Biochem J 111, 1-5.

Forsberg, C. W., Wyrick, P. B., Ward, J. B. \& Rogers, H. J. (1973). Effect of phosphate limitation on the morphology and wall composition of Bacillus licheniformis and its phosphoglucomutase-deficient mutants. J Bacteriol 113, 969-984.

Grant, W. D. (1979). Cell wall teichoic acid as a reserve phosphate source in Bacillus subtilis. J Bacteriol 137, 35-43.

Hughes, R. C. (1970). The cell wall of Bacillus licheniformis NCTC 6346. Biochem J 117, 431-439.

Janczura, E., Perkins, H. R. \& Rogers, H. J. (1961). Teichuronic acid: a mucopolysaccharide present in wall preparations from vegetative cells of Bacillus subtilis. Biochem J 80, 82-93.

Kruyssen, F. J., de Boer, W. R. \& Wouters, J. T. M. (1980). Effects of carbon source and growth rate on cell wall composition of Bacillus subtilis subsp. niger. J Bacteriol 144, 238-246.

Kunst, F., Ogasawara, N., Moszer, I. \& 148 other authors (1997). The complete genome sequence of the gram-positive bacterium Bacillus subtilis. Nature 390, 249-256.

Mauck, J. \& Glaser, L. (1970). Periplasmic nucleoside diphosphate sugar hydrolase from Bacillus subtilis. Biochemistry 9, 1140-1147.

Merchante, R., Pooley, H. M. \& Karamata, D. (1995). A periplasm in Bacillus subtilis. J Bacteriol 177, 6176-6183.

Moszer, I., Glaser, P. \& Danchin, A. (1995). SubtiList: a relational database for the Bacillus subtilis genome. Microbiology 141, 261-268.

Némoz, G., Prigent, A.-F. \& Pachéco, H. (1983). Analysis of cyclic nucleotide phosphodiesterase by isoelectric focusing coupled to a specific activity stain. Anal Biochem 133, 296-301.
Ordman, A. B. \& Kirkwood, S. (1977). UDP-glucose dehydrogenase kinetics and their mechanistic implications. Biochim Biophys Acta 481, 25-32.

Petit, C., Rigg, G. P., Pazzani, C., Smith, A., Sieberth, V., Stevens, M., Boulnois, G., Jann, K. \& Roberts, I. S. (1995). Region 2 of the Escherichia coli $\mathrm{K} 5$ capsule gene cluster encoding proteins for the biosynthesis of the K5 polysaccharide. Mol Microbiol 17, $611-620$.

Rosenberger, R. F. (1976). Control of teichoic and teichuronic acid biosynthesis in Bacillus subtilis $168 \mathrm{trp}^{-}$. Evidences for repression of enzyme synthesis and inhibition of enzyme activity. Biochim Biophys Acta 428, 516-524.

Soldo, B., Lazarevic, V., Pagni, M. \& Karamata, D. (1999). Teichuronic acid operon of Bacillus subtilis 168. Mol Microbiol 31, 795-805.

Strominger, J. L., Maxwell, E. S. \& Kalckar, H. M. (1957). Determination of UDPG and UTP by means of UDPG dehydrogenase. Methods Enzymol 3, 974-977.

Ujita, S. \& Kimura, K. (1982). Glucose-6-phosphate dehydrogenase, vegetative and spore Bacillus subtilis. Methods Enzymol 89, 258-261.

Wright, J. \& Heckel, J. E. (1975). The teichuronic acid of cell walls of Bacillus subtilis W23 grown in a chemostat under phosphate limitation. Biochem J 147, 187-189.

Zalitis, S. \& Feingold, D. S. (1969). Purification and properties of UDPG dehydrogenase from beef liver. Arch Biochem Biophys 132, $457-465$.

Received 4 June 1998; revised 25 January 1999; accepted 29 January 1999. 